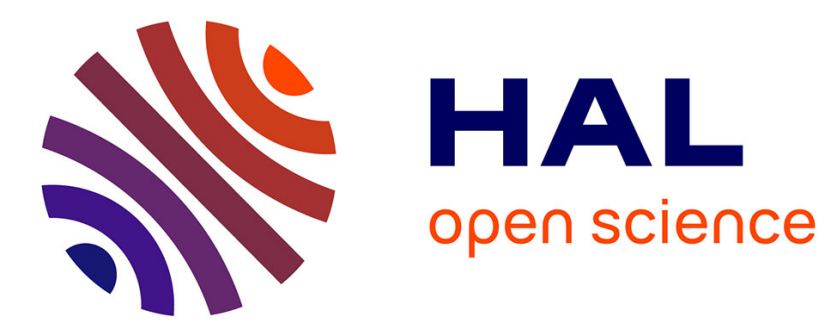

\title{
Quand les frontières du stérile et du non-stérile s'évanouissent
}

\author{
Josiane Tantchou, Marc-Eric Gruénais
}

\section{To cite this version:}

Josiane Tantchou, Marc-Eric Gruénais. Quand les frontières du stérile et du non-stérile s'évanouissent: De l'usage du bloc opératoire dans un contexte de crise du système de santé (Cameroun ). Revue d'Anthropologie des Connaissances, 2009, 10.3917/rac.008.0458 . hal-02441189

\section{HAL Id: hal-02441189 \\ https://hal.science/hal-02441189}

Submitted on 15 Jan 2020

HAL is a multi-disciplinary open access archive for the deposit and dissemination of scientific research documents, whether they are published or not. The documents may come from teaching and research institutions in France or abroad, or from public or private research centers.
L'archive ouverte pluridisciplinaire HAL, est destinée au dépôt et à la diffusion de documents scientifiques de niveau recherche, publiés ou non, émanant des établissements d'enseignement et de recherche français ou étrangers, des laboratoires publics ou privés. 


\title{
QUAND LES FRONTIÈRES DU STÉRILE ET DU NON-STÉRILE S'ÉVANOUISSENT
}

\author{
De l'usage du bloc opératoire dans \\ un contexte de crise du système de \\ santé (Cameroun)
}

Josiane Tantchou et Marc-Éric Grunénais

\begin{abstract}
RÉSUMÉ
Les travaux de recherches anthropologiques sur les espaces opératoires, la pratique chirurgicale ou le métier de chirurgien sont rares. Joan Cassel s'est intéressée à la personnalité des chirurgiens en rapport avec les spécificités de la chirurgie en tant que spécialisation. Marie-Christine Pouchelle (2003) a analysé le « toucher » à l'hôpital en insistant particulièrement sur la dimension chirurgicale. Pearl Katz a proposé une typologie du bloc opératoire en fonction des critères du stérile et du non-stérile qui y sont appliqués et a interrogé les rituels et les procédures techniques dans les blocs opératoires. Hirschauer (1991) s'est intéressé à l'acte chirurgical en tant que moment de rencontre de deux corps disciplinés (disciplined bodies) : le corps du patient (parcelled patient-body) et le " corps chirurgical ». Nous avons voulu poursuivre les réflexions de Katz (198I) mais surtout proposer une réflexion autour de la façon dont, dans un contexte spécifique qui est celui de crise du système de santé, le bloc opératoire et son équipement sont appropriés, son usage détourné ou renégocié. Nous montrons que le type d'appropriation du bloc opératoire dans ce contexte fait exploser les restrictions qui en font un espace confiné, stérile ; les limites du stérile et du non-stérile s'évanouissent, la pratique chirurgicale est dépouillée de son épaisseur, le bloc opératoire banalisé.
\end{abstract}

Loin de proposer des analyses définitives, cet article veut réorienter les débats sur les dysfonctionnements des systèmes de santé en Afrique. Pour ce faire, nous proposons d'approcher l'espace opératoire comme élément d'un système technologique : l'hôpital. Cette approche nous permettra de dépasser les orien- 
tations normatives courantes en anthropologie de la maladie, pour proposer une lecture du mode d'organisation et de fonctionnement des structures hospitalières en Afrique comme type spécifique d'appropriation d'un système technologique qu'il faut décrypter et analyser.

Mots Clés : Anthropologie de la santé, médecine, chirurgie, espace stérile, frontières, hôpital, système technologique, Cameroun, Afrique.

\section{INTRODUCTION}

Les travaux de recherches anthropologiques sur les espaces opératoires, la pratique chirurgicale ou le métier de chirurgien sont rares. Joan Cassel s'est par exemple intéressée à la personnalité des chirurgiens en la mettant en rapport avec les spécificités de la chirurgie en tant que spécialisation. Elle montre que, de sexe masculin pour la plupart (du moins pour ce qui concerne les États-Unis), les « traits » des chirurgiens ressemblent en plusieurs points à ceux des pilotes d'essais auxquels les chirurgiens se comparent par ailleurs. Comme Chuck Yeager, le célèbre pilote qui avait été le premier à franchir le mur du son, le chirurgien type n'a peur de rien ; il prend des risques, il est invulnérable, infatigable.

Dans cette même perspective, Marie-Christine Pouchelle (2003), analysant le « toucher » à l'hôpital en insistant particulièrement sur la dimension chirurgicale, a remarqué que les chirurgiens ont bien souvent pour modèle celui du guerrier impavide qui n'est, au moins en apparence, affecté par rien. C'est la violente intimité du contact relationnel qu'induit le geste chirurgical qui nécessite cette armure psychique, laquelle n'exclut pas le souci de l'autre, ni la compassion. Aussi « intouchables » que ces employés au contact quotidien des décédés considérés comme des manutentionnaires un peu dégoûtants, "morts 》 et contagieux comme les lépreux, les chirurgiens qui pénètrent dans les intimités inviolées du corps d'autrui le sont parce que « propres » et « purs » par excellence ; sont alors prohibés, parce que potentiellement contaminant pour eux, les contacts avec ceux-là qui ne sont pas stériles.

Pearl Katz a pour sa part proposé une typologie du bloc opératoire en fonction des critères du stérile et du non-stérile qui y sont appliqués et a interrogé les rituels et les procédures techniques dans les blocs opératoires. Elle montre ainsi que manifestement destinés à prévenir les infections, ces rituels et procédures techniques ont d'autres fonctions latentes. Ils établissent une différenciation des espaces, individus et instruments en stériles et non stériles; ils facilitent ou limitent l'autonomie d'action des personnes prenant part à l'intervention, renforçant de ce fait l'efficacité de l'équipe chirurgicale.

Hirschauer (199I) s'est enfin intéressé à l'acte chirurgical en tant que moment de rencontre de deux corps disciplinés (disciplined bodies) : le corps du patient (parcelled patient-body) et le « corps chirurgical ». Le corps du patient 
est préparé en sorte que seul l'organe ou l'espace à opérer soit accessible, d'où l'expression " parcelled patient-body ». Quant au « corps chirurgical », il fait référence au chirurgien, à son équipe, à tous les outils et équipements chirurgicaux qui le prolongent et renforcent son efficacité thérapeutique. Dans son analyse, Hirschauer (1991) arrive à démontrer que le corps du patient sert de modèle didactique que le chirurgien confronte au " corps anatomique » (anatomical body), le modèle esthétique incorporé pendant les leçons d'anatomie.

Nous avons voulu poursuivre les réflexions de Katz (198I), Hirschauer (1991), mais surtout proposer une réflexion autour de la façon dont, dans un contexte spécifique, le bloc opératoire et son équipement sont appropriés ; nous essayerons de montrer comment son usage est détourné ou renégocié dans ce contexte spécifique, qui est un contexte de crise du système de santé .

Les données ont été collectées dans le cadre d'un travail de recherche analysant l'impact du syndrome d'épuisement personnel du soignant (burn-out) sur la prise en charge des personnes vivant avec le $\mathrm{VIH} /$ sida. Nous montrons que le type d'appropriation du bloc opératoire dans ce contexte fait exploser les restrictions qui en font un espace confiné, stérile ; les limites du stérile et du non-stérile s'évanouissent, la pratique chirurgicale est dépouillée de son épaisseur, le bloc opératoire banalisé.

Loin de proposer des analyses définitives, cet article a la prétention de vouloir réorienter les débats sur les dysfonctionnements des systèmes de santé en Afrique. Pour ce faire, nous voulons approcher l'espace opératoire comme élément d'un système technologique : l'hôpital. Nous empruntons à Austeen et Headrick (1983, p. 168) la définition du système technologique :

« Le système technologique inclut un ensemble de matériaux couramment utilisés, des sources d'énergie, des types de travaux et d'organisation sociale, des outils, techniques et produits, des biens et des services. ॥

Approcher les technologies en tant que système permet de ne pas se limiter à l'étude des objets et éventuellement l'histoire de leur invention, leurs inventeurs. Cela permet de se pencher sur des ensembles, la façon dont ils sont liés entre eux, leurs origines, les transformations qu'ils subissent, leur interprétation, leur imbrication aux autres aspects de la société (Austeen et Headrick, 1983). Une pareille approche permettra de dépasser les orientations normatives courantes en anthropologie de la maladie, pour proposer une lecture du mode de (dys) fonctionnement des structures hospitalières en Afrique, comme type spécifique d'appropriation d'un système technologique, qu'il faut décrypter.

I La crise aiguë des systèmes de santé africains a été mise en évidence dans plusieurs travaux de recherches dont ceux de Chen (2004), Marchal et De Brouwere (2004), Narasimhan (2004), Delanyo (2005). Cette crise touche l'effectif, la qualité et la répartition des soignants. Voir Gruénais (200I) et Beyeme Ondoua (2002), notamment pour le Cameroun. 


\section{LE BLOC OPÉRATOIRE «ICI » : UN ENVIRONNEMENT PARTICULIER}

Le bloc opératoire est l'ensemble des bâtiments, des locaux et des équipements nécessaires aux opérations chirurgicales. II permet la prise en charge globale et individualisée des patients par une équipe interdisciplinaire (chirurgiens, anesthésistes, radiologues, gastro-entérologues, pneumologues, infirmières de bloc opératoire, aides-soignants, brancardiers...). De façon générale, le bloc opératoire est constitué au minimum d'une salle d'opération, le plus souvent de deux, voire une dizaine de salles dans les grands centres hospitaliers. La salle d'opération doit avoir des dimensions permettant de contenir un grand nombre d'équipements plus ou moins lourds et volumineux (négatoscopes, appareil délivrant de l'oxygène, électrocardiographe, matériel de réanimation, etc.), pour la plupart mobiles.

La salle d'opération est constituée de plusieurs pièces : un local où est installé le patient à opérer, une pièce pour le préparer pour l'intervention et un local (" scrubbing-room ») permettant aux chirurgiens et aux autres intervenants de se laver les mains en suivant une procédure bien spécifique et de se revêtir de vêtements stériles. La salle d'opération doit être équipée de prises électriques, de circuits d'alimentation (oxygène, protoxyde d'azote), de circuits d'aspiration, de tiroirs, de placards de rangement pour les instruments utilisés lors des opérations, etc.

Image I : un bloc opératoire « ici »

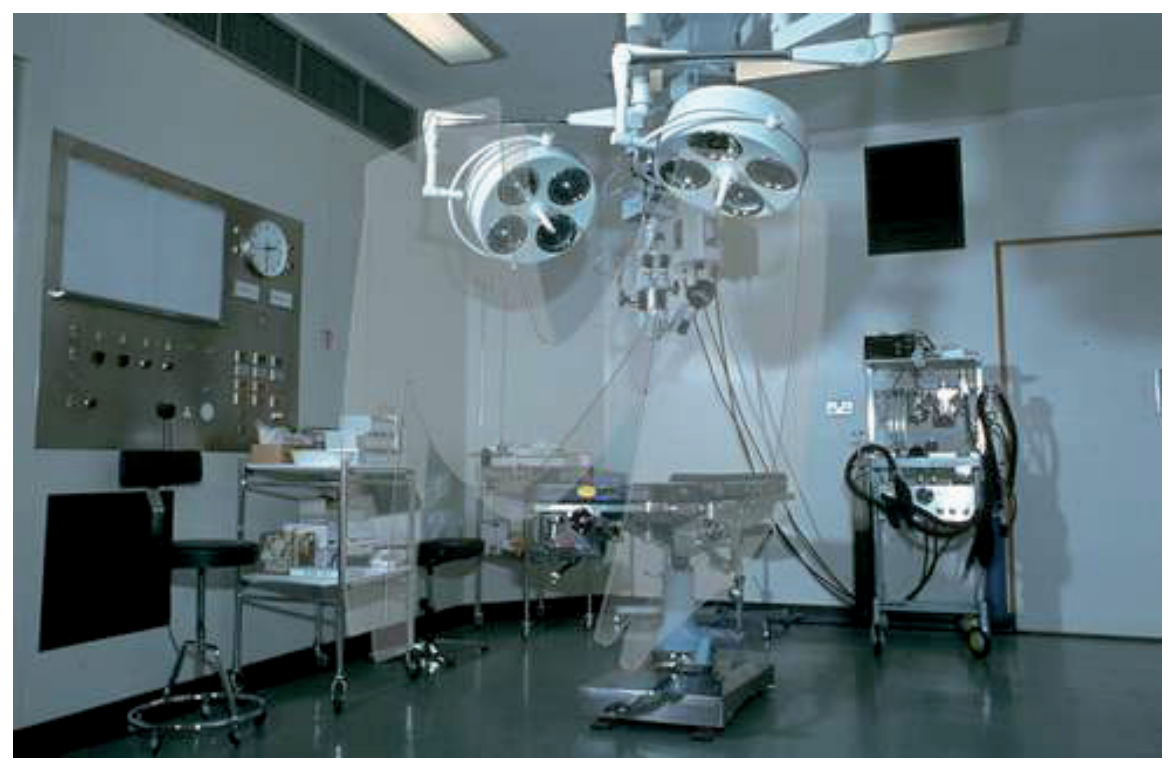

Source : Mediscan2853 
La première qualité du bloc opératoire est sa stérilité ; il doit être totalement indépendant du reste de l'hôpital et rendu étanche de celui-ci. Ses murs et sols doivent être lavables facilement, son écosystème maintenu à un niveau de contamination minimum par un nettoyage dont les rythmes préfixés seront scrupuleusement observés. La présence d'un système d'aération spécifique est nécessaire pour filtrer, stériliser et renouveler l'air du bloc opératoire. Des mesures restrictives participent à entretenir la propreté, la stérilité et prévenir la contamination du bloc opératoire. Ces restrictions imposent une gestion particulière des espaces. Ainsi, à mesure qu'on s'avance vers la salle d'opération, on passe des espaces propres à des espaces stériles (Katz, 198I ; Hirschauer, 1991), avec des restrictions de plus en plus sévères en termes d'accès et des exigences précises en matière de tenue vestimentaire (Hirschauer, 1991).

En fonction des critères du stérile et du non-stérile, Katz (198I) a proposé une typologie du bloc opératoire en quatre espaces : la « périphérie », « l'espace extérieur », le « milieu » et " l'espace intérieur ». La « périphérie » est l'espace le moins propre. Pour y accéder on doit se revêtir d'une blouse blanche permettant l'identification en tant que soignant, membre de l'équipe médicale. Dans I'hôpital qu'elle étudie, les cabinets d'anesthésistes, un laboratoire, un dressing pour hommes et femmes, des salons pour les infirmiers et les médecins y sont situés. Séparé de la " périphérie » par une porte coulissante, c'est à "l'extérieur » que sont affichés les programmes d'interventions, $y$ attendent aussi les patients qui doivent être opérés. C'est aussi à « l'extérieur » que sont situées les salles d'observation, là où les patients sont transférés après l'intervention chirurgicale. L'entrée dans cet espace est réservée aux malades et aux membres de l'équipe médicale ayant revêtu une tunique et un pantalon de couleur bleue ou verte. Une infirmière s'assure que seules les personnes autorisées y accèdent. Le milieu » est composé d'espaces stériles rattachés à l'extérieur et au bloc opératoire par des portes. Chacun de ces espaces dispose d'autoclaves, de récipients renfermant les instruments de chirurgie, des draps et des serviettes. Pour y accéder, on doit porter des chaussures spécifiques, un masque (bleu ou vert) recouvrant le nez et la bouche ; les cheveux doivent également être recouverts. Les salles d'opérations sont situées dans « l'espace intérieur ». Pearl Katz (198I) résume cette structure interne de l'espace opératoire en proposant le schéma ci-après : 
Schéma I : L'espace opératoire d'après la catégorisation de Katz (198I)

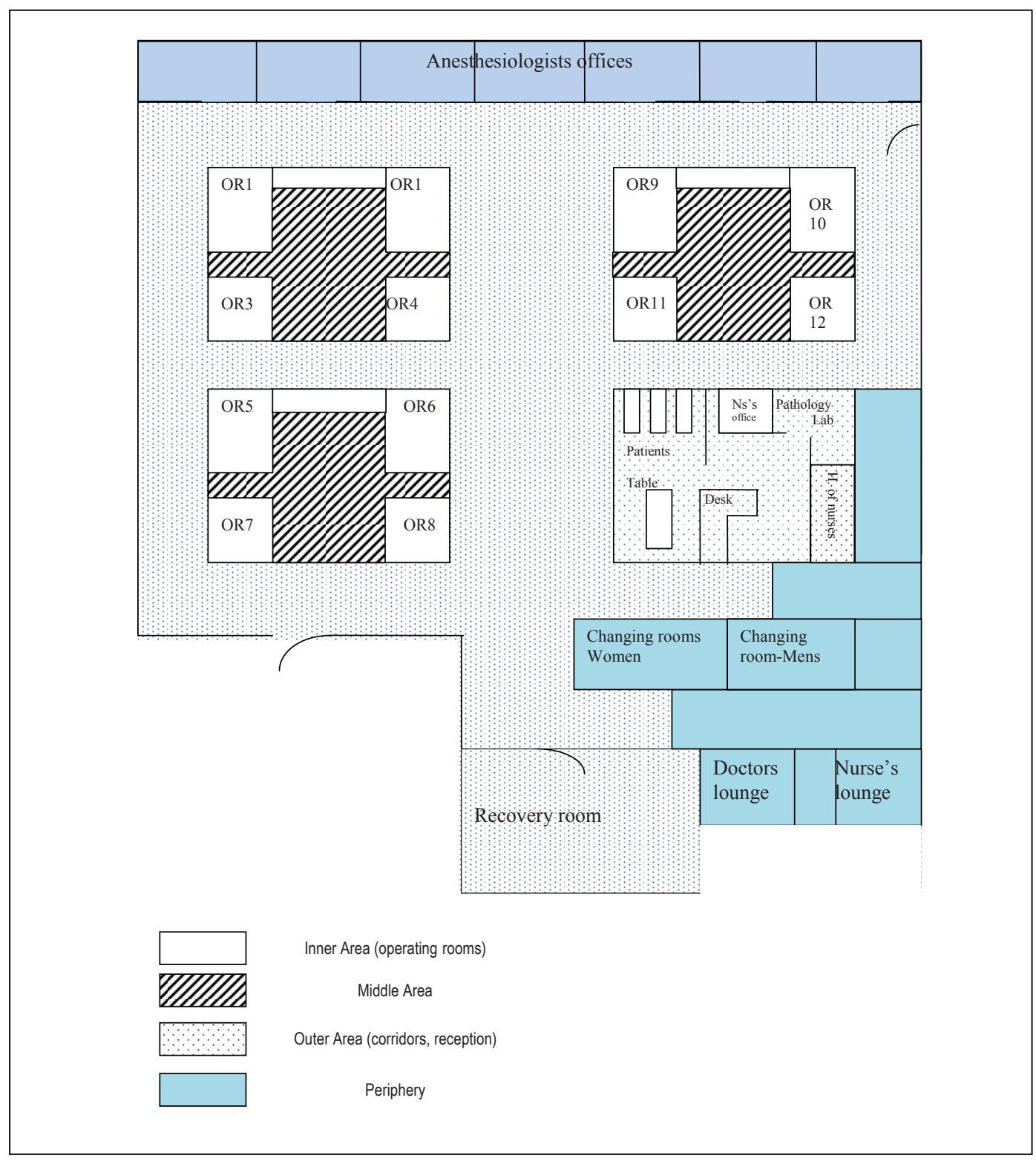

Soulignons que les salles d'opération ne sont pas homogènes. Dès qu'on n'est plus au contact direct de la table d'opération ou de celle de l'instrumentiste, on est en territoire propre mais non stérile. Ainsi, l'espace des anesthésistes est le plus souvent séparé de celui des chirurgiens par un grand " champ » vertical situé au niveau du cou du patient (ce champ est inexistant dans le contexte étudié). Chez les anesthésistes, on est en territoire propre, mais non stérile, donc relativement " sale » (Pouchelle, 2003). L'opéré, quant à lui, peut être potentiellement contaminant ( $\mathrm{VIH} /$ sida, hépatites, autres maladies infectieuses) et la chirurgie pratiquée plus ou moins « sale » (chirurgie digestive des tuyaux, orl, chirurgie des poumons). Ce qui a touché le corps des patients infectés ou non doit donc être systématiquement jeté dans un sac poubelle spécifique 
consacré aux " déchets infectieux », faisant l'objet d'un traitement spécial. Le risque de contagion fonctionne donc dans les deux sens. C'est la raison pour laquelle dans le bloc opératoire, la différenciation des espaces suscite des comportements contrôlés : on n'y touche pas et on n'y bouge pas n'importe comment (Pouchelle, 2003).

C'est familiarisé à ces représentations que nous nous sommes rendue sur le terrain. Venons-en au contexte étudié ; nous allons présenter la structure interne du bloc opératoire et son emplacement au sein de l'hôpital. Emplacement et structure interne qui font exploser la typologie proposée par Pearl Katz (I98I), mettant en évidence un type spécifique d'appropriation du système technologique qu'est l'hôpital. Mais avant, une note méthodologique nous permettra de montrer comment confrontée à la réalité d'un bloc opératoire dans ce contexte particulier, les images qui avaient nourri notre imagination, nos représentations ont été violemment remises en question.

\section{LE BLOC OPÉRATOIRE « LÀ-BAS » ${ }^{2}:$ UN ESPACE BANALISÉ}

\section{Le contact avec le bloc opératoire, une expérience déroutante : est-ce bien un bloc opératoire?}

Notre premier contact avec ce bloc opératoire a été déroutant ; son équipement rudimentaire et sale donnait à penser à un local accueillant les équipements en panne ou usagés, plus qu'à l'environnement propre et stérile auquel notre imagination avait été familiarisée. Les soignants n'hésitaient pas à remarquer :

« Si on respectait les normes, notre bloc opératoire serait fermé depuis très longtemps » (major du service).

Nos principales interrogations étaient alors les suivantes : qui se faisait opérer dans ces salles ? Pour quelles raisons ? Pour quelles maladies ? Ces questions étaient d'autant plus justifiées qu'une patiente à laquelle le chirurgien faisait remarquer qu'heureusement il était là pour sauver la vie des membres de sa communauté, lui a dédaigneusement répondu :

« Moi, dans votre truc là ? Jamais. Je vais à Dubaï. »

2 Au Cameroun, le secteur santé s'articule en trois sous-secteurs : public, privé, traditionnel. Le secteur public (qui nous intéresse) est organisé en trois niveaux : central, intermédiaire et périphérique. Le niveau central qui élabore les politiques, les normes, les stratégies nationales est doté de six hôpitaux de référence nationale. Le niveau intermédiaire représenté par des délégations provinciales de la santé publique traduit en programmes la politique élaborée au niveau central. Les structures sanitaires à ce niveau sont les hôpitaux provinciaux. Le district de santé constitue le niveau périphérique. II est subdivisé en " aires de santé » et est doté de deux types d'établissement de soins : l'hôpital de district et le centre de santé intégré (Gruénais, 200 I ; Beyeme Ondoua, 2002). Les données présentées ici ont été collectées dans un hôpital provincial. 
Puis, comment pouvait-on respecter les règles d'asepsie quand l'environnement ne s'y prêtait pas ? Et comment expliquer qu'en dépit de cet état de chose, les cas d'infections postopératoires ne soient pas courants ?' Ainsi, sommes-nous naïvement arrivés à nous demander si les frontières du propre et du sale, du stérile et du non-stérile et toutes les procédures visant à confiner et stériliser le bloc opératoire ne relevaient pas finalement d'une majoration de risques, d'une exigence de « sécurité maximale » (Vayre, 2000), en rapport avec la responsabilité juridique du chirurgien d'une part et d'autre part, l'obsession du risque et la nécessité de dispositifs assurant à tous les niveaux un rapprochement du risque zéro, dont on a fait une des caractéristiques des sociétés occidentales contemporaines (Beck, 2003).

Image 2 : Vue de la salle de stérilisation

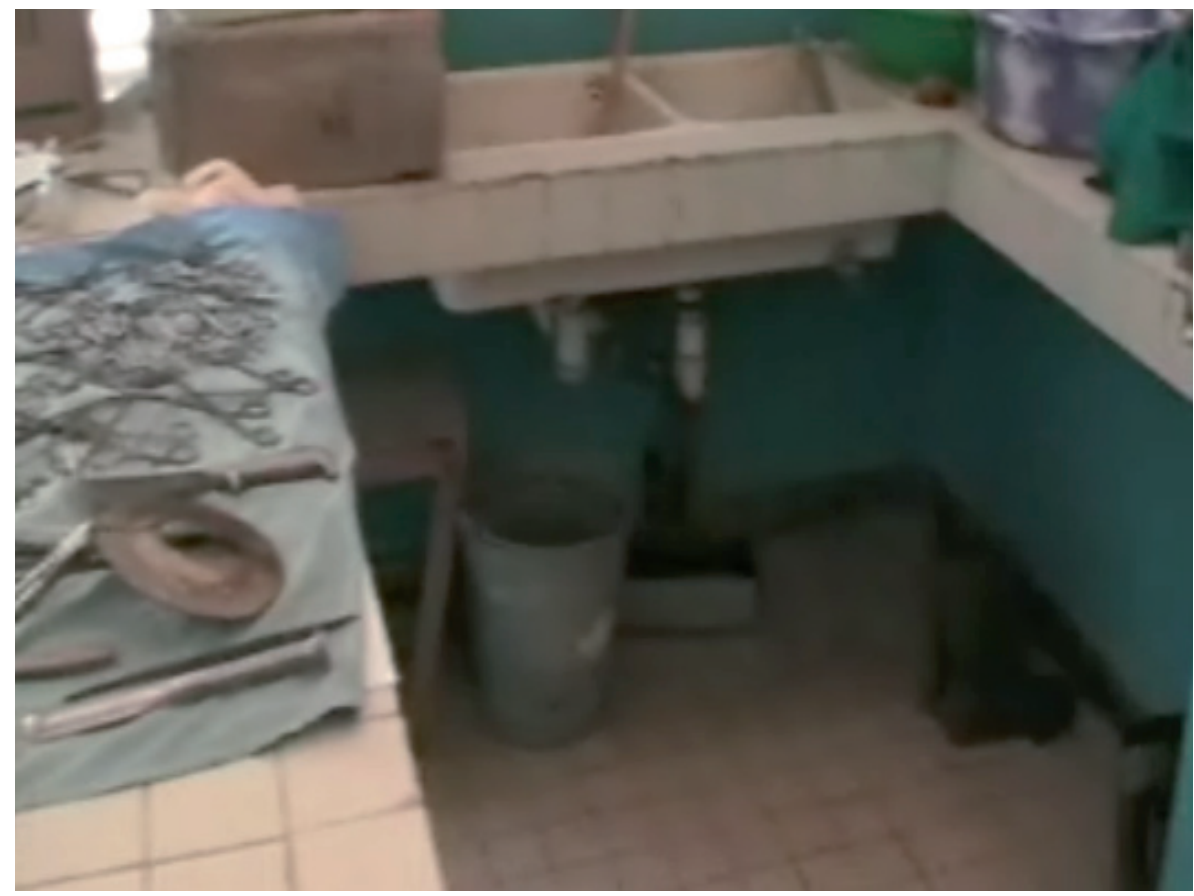

Avec l'accord de l'administration de hôpital et celui du chirurgien chef du service, nous avons assisté ${ }^{4}$ à toutes les opérations chirurgicales effectuées durant notre séjour ; nous avons suivi l'équipe chirurgicale lors des visites médicales quotidiennes (rondes hospitalières), assisté aux consultations du chirurgien chef du service.

3 Source : entretien avec le chirurgien, registre du service.

4 Comme déjà mentionné, les données présentées dans ce texte sont tirées d'un travail de recherche intitulé : « Burnout et prise en charge des personnes vivant avec le VIH/sida au Cameroun ». Pendant cinq mois, nous avons procédé à des observations et réalisé des entretiens approfondis dans les services de deux hôpitaux de niveau intermédiaire du Grand Nord du Cameroun. Les observations étaient conduites de $3 \mathrm{~h}$ à $8 \mathrm{~h}$ par jour dans les services prenant en charge les personnes vivant avec le $\mathrm{VIH} /$ sida $(\mathrm{PVVIH})$. Elles duraient le temps nécessaire pour arriver à un niveau de redondance dans les données (saturation des données). Les observations étaient répertoriées quotidiennement dans un « journal de bord » où figuraient également les ébauches d'analyse. 
Image 3 : Salle d'opération après l'acte chirurgical

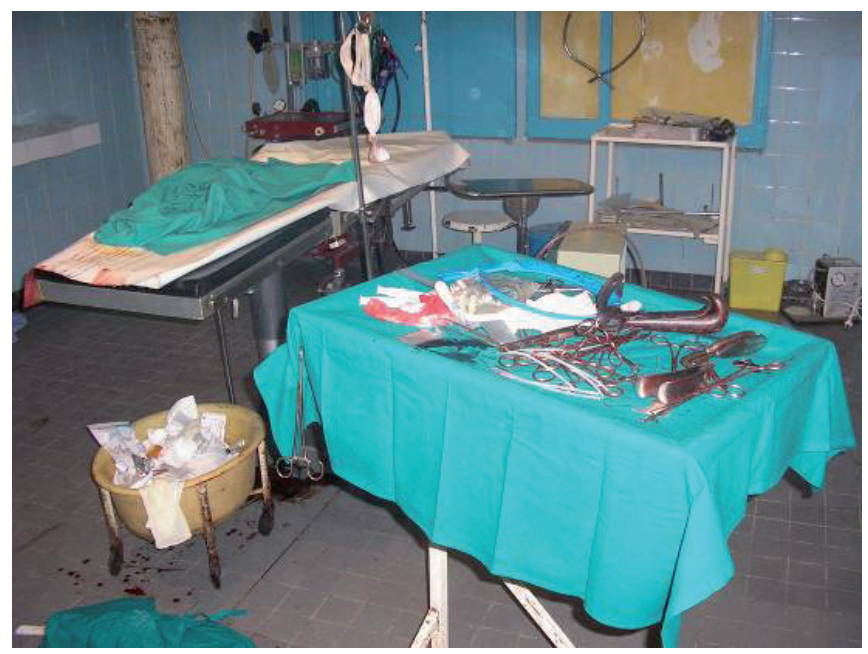

Nous n'avons pas assisté à la préparation des patients par l'anesthésiste avant l'intervention chirurgicale. Contrairement à ce qui se passe dans certains hôpitaux occidentaux où les patients sont préparés par l'anesthésiste dans le bloc opératoire, ici, la préparation des patients se déroule dans les salles d'hospitalisation. Celles-ci sont de façon générale partagées par quatre ou cinq malades. Les patients, comme l'a aussi noté Marie-Christine Pouchelle (2003), sont terriblement « touchants ». Leur regard, soulignait-elle encore, est « gluant » : « surtout ne pas rencontrer leurs yeux : de quoi prendre ses jambes à son cou ॥. Dans le cas précis, les regards appelaient la fuite ou l'implication, ce qui conduisait nécessairement à une négociation constante de notre positionnement, entre distance, proximité et implication. Pour des raisons de pudeur, et surtout pour ne pas dépasser les limites de l'implication (émotionnelle), nous avons choisi d'attendre ou de précéder les patients, en compagnie du chirurgien et de l'équipe chirurgicale, dans la salle d'opération.

\section{Dans le bloc opératoire : qui suis-je au juste?}

Notre position dans le bloc opératoire était ambiguë. Nous avions l'obligation de porter une blouse blanche, une calotte et un masque mais nous n'étions équipée ni de gants, ni de chaussures spécifiques. Cela nous obligeait, pour limiter l'exposition aux liquides sanguins et réduire d'une certaine façon les risques d'accidents d'exposition au sang (AES), à rester à une certaine distance de la table d'opération. Par contre, il arrivait que des équipes médicales européennes soient en visite dans l'hôpital. Le gynécologue exigeait alors que les infirmiers (européens) voulant assister à une intervention, soient revêtus d'une tenue de bloc opératoire. 
Image 4 : L'exigence de tenue de bloc pour le personnel étranger ${ }^{5}$

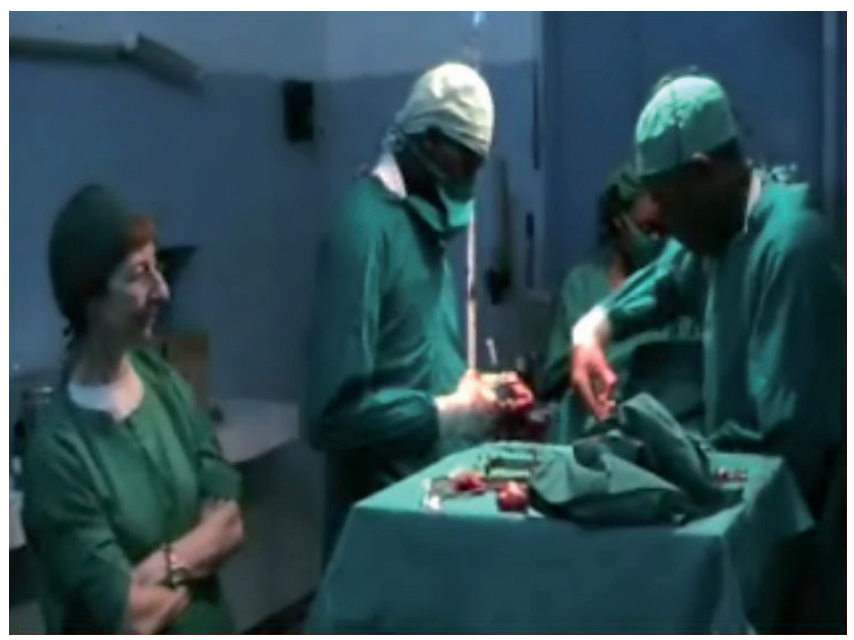

Or le personnel du service d'accueil ne respectait pas nécessairement cette indication. Ainsi, alors que le gynécologue procédait à l'extraction d'une tumeur maligne, la porte du bloc opératoire s'est brusquement ouverte devant son confrère (européen) ; de passage dans la ville, il souhaitait le rencontrer. Le gynécologue lui a fait remarquer qu'il n'était pas en tenue de bloc opératoire. Ce à quoi le confrère a répondu qu'il l'avait souligné au personnel à l'accueil, lesquels ont affirmé que ce n'était pas un problème. II s'est retiré quelques minutes plus tard après avoir pris un rendez-vous.

L’idée était de procéder à une observation non participante. Cependant, pour diverses raisons dont quelques-unes seront énoncées dans la suite du texte, nous avons de temps à autre été invitée à servir gants, pansements, désinfectant, à ouvrir les cuves renfermant les vêtements stériles au chirurgien ou à son aide, à débrancher un cordon électrique, à répondre au téléphone, ou à rapprocher le téléphone portable du chirurgien de son oreille, etc.

\section{Au bloc opératoire : les angoisses de l'observateur}

L'équipe chirurgicale était en moyenne constituée de trois, parfois deux personnes. La position respective de ses membres permettait d'avoir une vue de l'ensemble des acteurs. N'étant ni sous anesthésie comme le patient, ni obligée de nous concentrer sur l'acte chirurgical, nous pouvions librement observer les mouvements des uns et des autres, et écouter attentivement les discours. Nous nous tenions tantôt en face du chirurgien, en biais au niveau des pieds du patient ou à son chevet. Cette première position était une grande source d'angoisse ; angoisse de bloquer involontairement un mouvement et d'empêcher le chirurgien de saisir la bonne pince, le bon ciseau au bon moment ; angoisse d'effectuer un geste qui aurait heurté sa main, dévié la trajectoire de son bistouri et causé

5 Impossible de trouver un masque pour compléter la tenue de bloc ! 
un dommage irréversible. En outre, de cette position, nous avions le sentiment d'être happée par l'intervention chirurgicale, captivée par le mouvement, l'agilité, la précision des doigts du chirurgien.

Au chevet du patient, nous devions partager l'espace avec l'anesthésiste. Nous nous tenions à sa droite ou à sa gauche. Dans les deux cas, nous avions une vision assez réduite de la progression du « corps chirurgical » (un linge de couleur verte établissait une séparation entre la tête du patient et le champ opératoire). L'anesthésiste devait en outre régulièrement vérifier le pouls du patient, le débit des narcotiques coulant à travers une perfusion dont l'aiguille était insérée dans son avant-bras. Dès lors, pour ne pas entraver ses mouvements nous devions constamment changer de position, ce qui était aussi une grande source d'appréhension : crainte de distraire et déconcentrer le chirurgien ou son aide, de heurter un cordon électrique, de bousculer et/ou de faire tomber un objet, etc.

Nous mettons ces angoisses en rapport avec l'image que nous nous étions forgée du bloc opératoire : espace strictement stérile et discipliné, où le moindre mouvement, le moindre courant d'air, le moindre incident pouvait s'avérer fatal. L'aspect et le mode de fonctionnement du bloc opératoire étudié nous amèneront à prendre conscience de ces représentations et à les interroger. C'est pour ces différentes raisons que nous avons privilégié la position au niveau des pieds du patient, tantôt à son pied gauche, tantôt à son pied droit. De là, nous avions une vision totale de l'ensemble de l'équipe, nous pouvions aisément suivre la progression de l'intervention chirurgicale et écouter les discussions.

Image 5 : Aux pieds du patient, tantôt à gauche, tantôt à droite

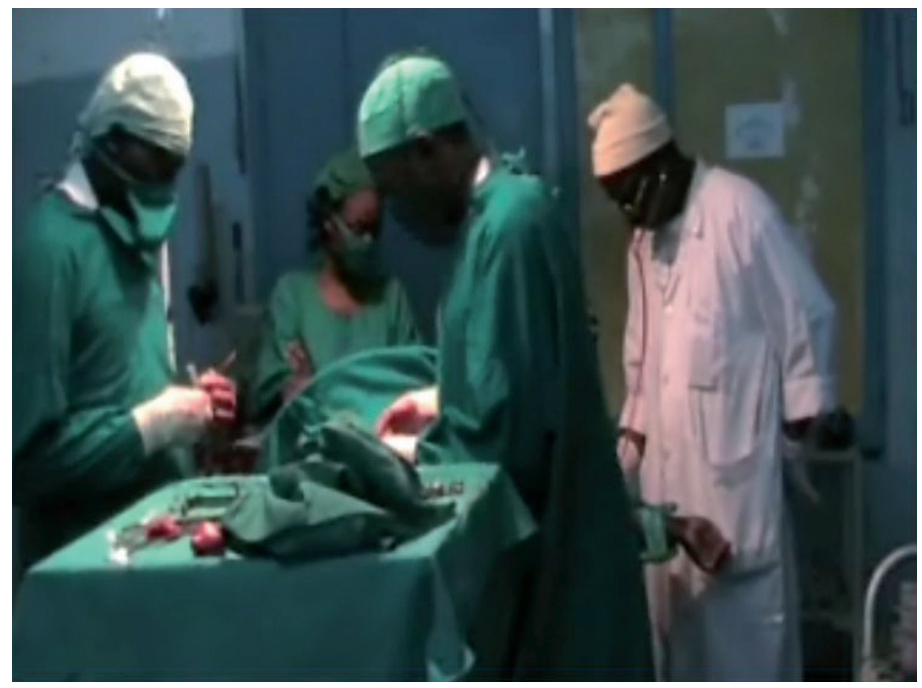

Les observations faites dans le bloc opératoire ont été complétées par des entretiens de type formel et informel réalisés avec l'équipe chirurgicale. Les premiers se déroulaient spontanément entre deux consultations, pendant la pause et quelquefois pendant l'intervention chirurgicale. Cela participait une 
fois encore à déconstruire les représentations que nous avions d'un bloc opératoire : espace où la parole était en suspension, les dialogues limités aux indications, précisions et intimations du chirurgien. Les entretiens informels n'étaient pas enregistrés. II s'agissait surtout de revenir sur certains points qui n'avaient pas été abordés pendant les entretiens formels, ou qui nécessitaient des précisions. Les entretiens formels quant à eux étaient préparés à l'avance. Nous prenions rendez-vous avec l'informateur ; sur un guide d'entretien, nous avions énoncé les points à aborder.

De façon générale, les entretiens se déroulaient au sein de la formation sanitaire ; ils étaient enregistrés (avec l'accord de l'informateur) et transcrits. Chaque transcription était précédée d'un certain nombre d'observations : l'âge de l'informateur, circonstances dans lesquelles l'entretien a été accordé, nombre et type de visites reçus pendant l'entretien. Venons-en à présent au bloc opératoire lui-même. Quel emplacement au sein de l'hôpital ? Quelle structure interne?

\section{Emplacement et structure interne du bloc opératoire}

Le pavillon opératoire étudié est situé au cœur de l'hôpital (Schéma 2), entre le service de médecine interne, le service de gynécologie-obstétrique et la direction de l'hôpital. II n'est donc isolé ni physiquement, ni par son architecture, du reste de l'hôpital. Un gynécologue, un chirurgien et un urologue (chef du service suivi pendant les enquêtes) utilisent l'espace opératoire. Les opérations principalement réalisées concernent les tumeurs bénignes, les tumeurs prostatiques, les grossesses extra-utérines ; hystérectomies, laparotomies, césariennes, amputations y sont également pratiquées.

Schéma 2 : Le bloc opératoire dans l'hôpital

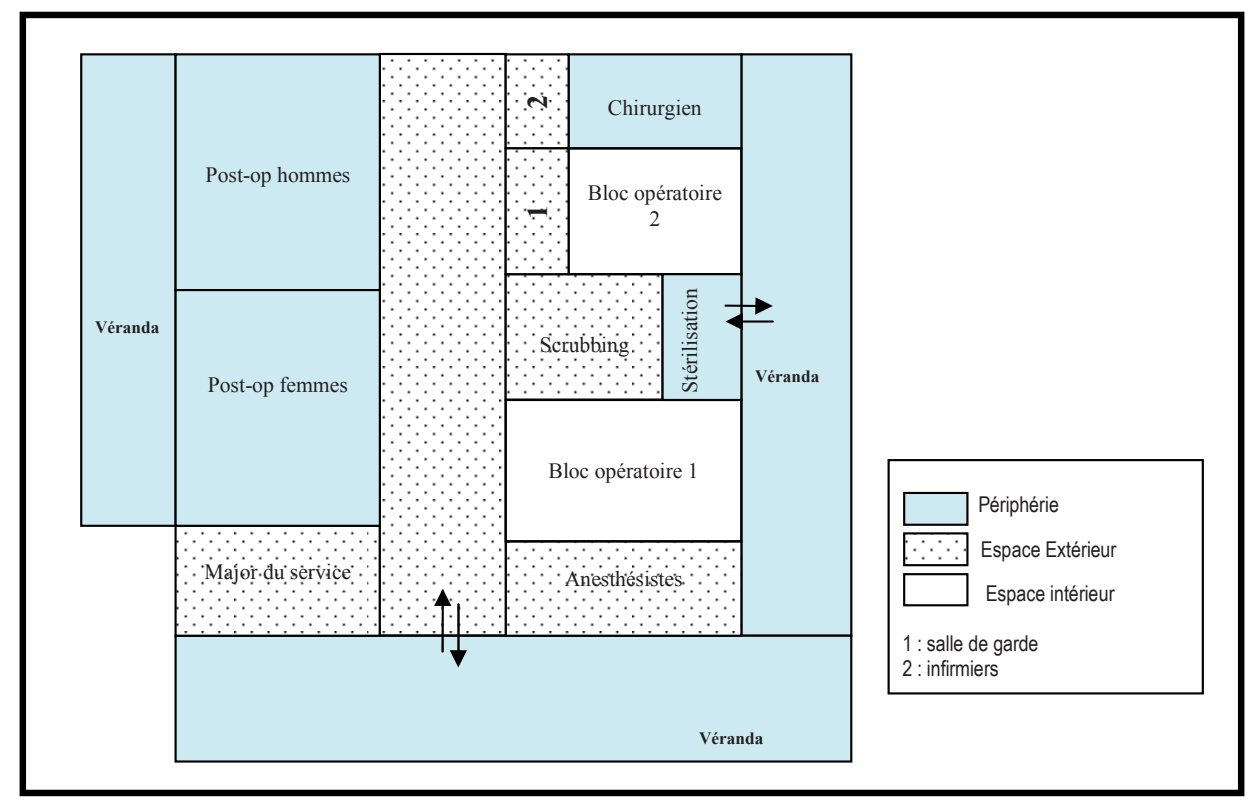


On accède au bloc par deux portes à battants auxquelles sont associées deux vérandas. La première porte s'ouvre sur la salle de stérilisation. Sa véranda mène au bureau du chirurgien et à une cour qui sépare le bloc opératoire du pavillon dit " chirurgie salle $»^{6}$. C'est par cette porte que le chirurgien quitte habituellement le service. L'intervention ayant souvent été longue, cette sortie lui permet entre autres d'échapper aux questions des membres de la famille du patient ${ }^{7}$.

Schéma 3 : Plan simplifié du bloc opératoire

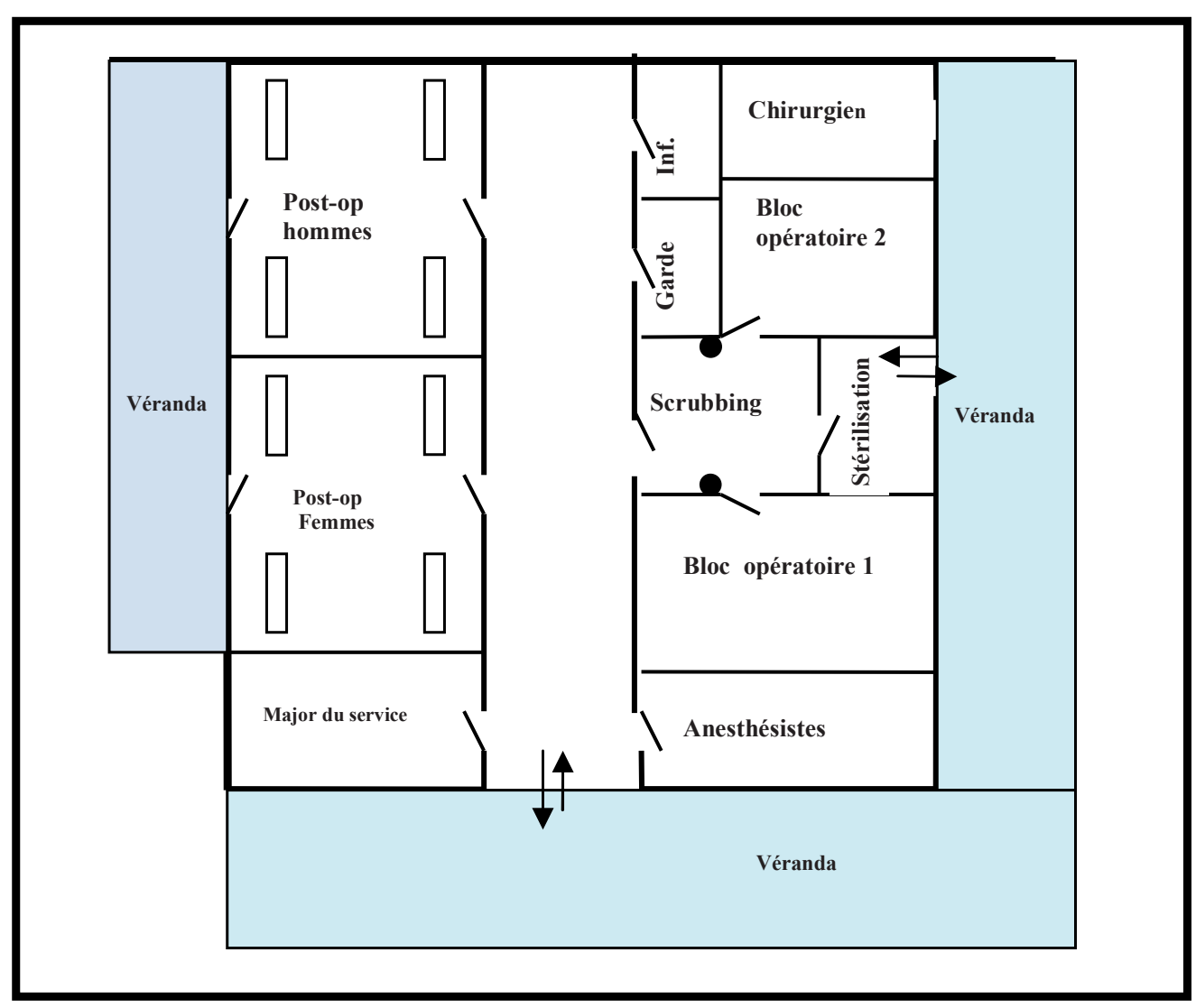

La deuxième véranda est équipée de bancs sur lesquels s'installent les soignants aux heures creuses ou de pause ; c'est aussi là que les membres de la famille du patient attendent la fin de l'opération. La porte associée à cette

6 Après trois jours en observation en salle d'hospitalisation postopératoire, les patients doivent en principe y être transférés pour la suite de leur convalescence.

7 II faut rappeler que les chirurgiens ne sont pas toujours plus à l'aise avec les malades. MarieChristine Pouchelle (2003) indique que le bureau du chirurgien chef d'un hôpital de la rue Tolbiac, à Paris, déplacé hors de la capitale en 1977, comportait une issue particulière qui donnait directement sur la rue. Cette issue lui permettait de sortir de son bureau sans courir le risque d'être accroché par des malades ou des familles par définition envahissants ; il pouvait donc quitter son bureau sans se « faire bouffer » par les malades. Nous avons déjà évoqué leur regard « touchant », " gluant ». II faut rappeler que le manque de tact des hommes de l'art, comme l'a encore souligné Pouchelle (2003), appartient aux stéréotypes de la profession. Certains se montrent si directs qu'ils en sont grossiers, d'autres sont dans l'évitement. Chez d'autres encore, on pourrait être tenté de penser à une phobie du contact relationnel proportionnelle à la violente intimité qu'induit le geste chirurgical. 
véranda est la plus utilisée. Elle s'ouvre sur un couloir non éclairé, donnant une atmosphère lugubre à l'intérieur du bâtiment. Sur la gauche du couloir sont situés : le bureau du major du service et deux salles destinées aux hospitalisations postopératoires; deux portes à battants facilitent le transfert des patients à opérer des salles d'hospitalisation vers les salles d'opération et vice-versa. Leur usage est réservé aux soignants. Toujours associées à ces salles, deux autres portes donnant sur une grande cour qui sépare le bloc opératoire du pavillon dit de haut standing. Ces passages sont à l'usage des patients, de leurs accompagnants et des visiteurs. Sur le même couloir à droite, se trouvent une salle de garde, un cabinet réservé aux anesthésistes, un cabinet réservé aux infirmiers et entre ces deux pièces, une porte s'ouvre sur le « scrubbing room ».

Le « scrubbing room » est équipé de deux lavabos à la propreté douteuse et d'un placard métallique délabré. C'est dans ces lavabos que les chirurgiens doivent se laver les mains selon une procédure précise, avant de s'introduire dans les salles d'opération. Ce rituel dit « scrubbing » (d'où l'expression « scrubbing-room ») serait, selon Pearl Katz (198I), l'un des plus importants de la procédure chirurgicale ${ }^{8}$. II marque le début du processus par lequel le chirurgien et son aide vont se transformer en fractions du « corps chirurgical » (Hirschauer, 199|). De part et d'autre du "scrubbing room », à quelques centimètres des lavabos, deux autres portes à battants s'ouvrent sur les deux salles d'opération. En suivant fidèlement la typologie proposée par Pearl Katz (I98I), le bloc opératoire étudié se présentera ainsi :

Schéma 4 : Espace opératoire étudié suivant la catégorisation de Katz (I98I)

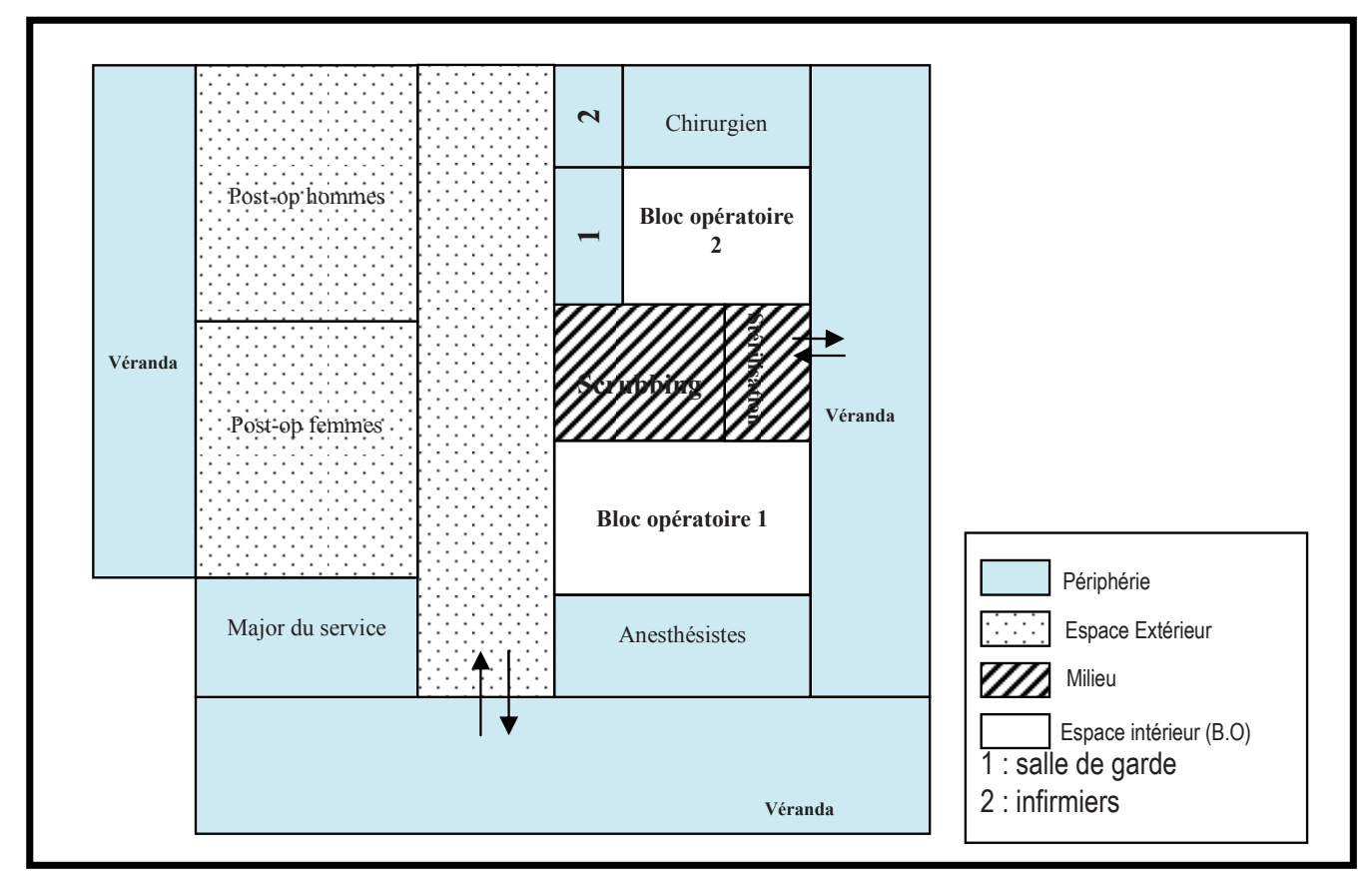

8 Voir Katz (198I) et Hirschauer (199I) pour une description de ce « rituel » dit « scrubbing ». 
Or le type d'appropriation de l'espace opératoire dans ce contexte rend une pareille structuration de l'espace inopérante, principalement à cause de l'inapplicabilité des restrictions en matière d'entrée et de sortie, donc de maintien de la stérilité de l'air et des espaces.

\section{Frontières poreuses : banalisation de l'espace opératoire}

Comme indiqué, trois spécialistes utilisent le bloc opératoire. En plus de leurs fonctions de spécialistes, ils assurent les consultations de médecine interne. De façon générale, l'équipe chirurgicale se compose du chirurgien, de son assistant et d'un anesthésiste. Les bénévoles ${ }^{9}$ qui sont affectés au pavillon opératoire sans attributions précises, assistent aux interventions de leur choix. Les déplacements dans l'espace opératoire ne sont pas contrôlés ; les restrictions relatives à l'accès au bloc opératoire ne peuvent donc être opérationnelles. Un agent d'entretien assure la propreté des espaces avant et après les actes chirurgicaux. II assure également la stérilisation des instruments. Entre deux coups de serpillière ou de balai, il doit surveiller les autoclaves servant à stériliser les instruments. Faisant aussi fonction de brancardier, il sera invité à interrompre ce travail ou le désherbage des alentours du pavillon et, sans précautions particulières, s'introduira dans la salle d'opération pour assurer le transfert d'un patient vers les chambres d'hospitalisation postopératoires, ou préparer la salle d'opération pour l'intervention suivante (il s'agira simplement de nettoyer le sol et la table d'opération à l'aide d'un mélange d'eau de javel). Sans prendre de mesures de précautions particulières, le même agent d'entretien introduira des enfants dans le local de stérilisation pour assurer le pansement de glands fraîchement circoncis.

À cause de la précarisation des fonctions soignantes dont l'absentéisme (Jaffré et Sardan, 200I ; Vidal et al., 2005) est une conséquence majeure, il est difficile d'élaborer un planning des équipes. Le chirurgien travaillera donc avec les soignants présents ; il sera même parfois obligé de faire venir d'urgence un anesthésiste de repos. En outre, du fait de la pauvreté des patients, les interventions ne peuvent être programmées à l'avance. En effet, le coût moyen d'une intervention dépasse largement les revenus de la plupart des patients. Par conséquent, une fois que le diagnostic a été posé, ceux-ci doivent prendre le temps de rassembler le montant nécessaire pour s'acquitter du coût de l'in-

9 Ce sont des personnels paramédicaux formés dans les différentes écoles publiques ou privées que compte le pays. Le ministère de la Santé publique ne recrutant plus de personnel (mis à part les médecins), pour « ne pas perdre la main », à leur sortie d'école, ces élèves se font recruter dans les hôpitaux et centres de santé en tant que « stagiaires »; de fait, ils sont « bénévoles ». Ils ont la même charge de travail que le reste du personnel et ne reçoivent aucune rémunération. La carence en ressources humaines en santé rend leur présence essentielle pour assurer le fonctionnement de certains services dans lesquels leur effectif est parfois nettement supérieur à celui des soignants statutaires. 
tervention. Au terme d'une consultation, si le patient affirme être capable de supporter ce coût, ou lorsqu'il revient après avoir réuni le coût, l'intervention chirurgicale a lieu dans la journée ou 24 heures plus tard.

Le chirurgien, après avoir prescrit certains examens, envoie le patient auprès de l'anesthésiste. Celui-ci lui prépare une ordonnance avec les éléments indispensables pour l'intervention (fil et aiguilles de suture, bistouri, narcotiques, perfusion, catlon, antiseptique, gants de chirurgien, etc.). Les patients et/ou les accompagnants se rendent alors à la pharmacie de l'hôpital pour acheter les produits prescrits, et au laboratoire pour le prélèvement de substances biologiques essentielles pour réaliser les examens demandés. Lorsque le processus est achevé et le rendu des examens effectué, l'anesthésiste envoie un message au chirurgien, lui demandant s'il peut procéder à la préparation du patient. Le chirurgien avertit alors les personnes attendant une consultation de son interruption imminente. Quand il a fini de préparer le patient, l'anesthésiste envoie de nouveau un message au chirurgien. Celui-ci se débarrasse de sa blouse blanche, se déshabille pour revêtir un pantalon, une tunique verte, une paire de bottes en plastique et une calotte vertes. Avec une sacoche et son téléphone portable, il se dirige vers la salle d'opération. Comme mentionné, il travaillera avec les membres de l'équipe soignante présents. Cependant, il arrive régulièrement qu'un anesthésiste ou un infirmier arrivant quelques instants après le début de l'intervention, par curiosité, ou pour se rendre utile, sans retenue particulière, s'introduise dans la salle d'opération. Dans le contexte de précarité caractéristique de la fonction soignante en Afrique, cela peut d'ailleurs être avantageux. Le chirurgien doit se donner les moyens de « motiver » son équipe et de se la « rendre fidèle ». Ainsi, le coût de l'intervention prévoit une rémunération pour le chirurgien et ses aides ; assister à une opération chirurgicale peut donc donner lieu à une « motivation », " l'argent de la bière », ou « l'argent du taxi », etc. Les bénévoles qui n'ont pour leur part aucune obligation en termes d'heures de service, et qui ne sont pas nécessairement « motivés », entrent et quittent la salle d'opération à leur guise.

L'hôpital ne fournit ni masque, ni « tenue de bloc » aux soignants. Ils y accèdent donc de façon générale en blouse blanche avec, suivant les cas, un masque et une calotte dont l'usage unique n'est dans ce contexte pas souvent respecté. Cet état de choses rend caduques toutes éventuelles restrictions quant à la tenue vestimentaire. En effet, s'il faut se mettre en « tenue de bloc », seuls le chirurgien et éventuellement son assistant pourront y avoir accès.

Les patients en attente d'une opération ou faisant l'objet d'un suivi postopératoire sont, de façon générale, accompagnés d'un ou plusieurs membres de leur famille. Ces accompagnants font en principe fonction d'aide-soignant. Ils s'occupent de la toilette, des repas, de l'administration des médicaments... de " leurs » patients. L'équipement du service ne prévoyant aucun système d'hébergement pour eux, ils étendent des nattes au pied des lits de malades pour $y$ passer la nuit. Le personnel se plaint régulièrement du non-respect des instruc- 
tions de l'équipe médicale par les accompagnants. Non seulement ils doivent se retirer au moment où l'équipe médicale commence la ronde, mais également faciliter son déplacement à l'intérieur des salles d'hospitalisation. C'est-à-dire débarrasser le sol des nattes à l'aube, s'arranger pour que les patients prennent leur petit déjeuner et pour que la vaisselle soit faite et les assiettes convenablement rangées avant les visites médicales, etc. Or, régulièrement, les soignants doivent piétiner les nattes, passer entre les récipients isothermes, les assiettes malpropres et/ou à moitié pleines. Cet état de chose est régulièrement à l'origine de tensions entre les accompagnants et l'équipe médicale, laquelle doit constamment leur intimer l'ordre de débarrasser le sol de " tout ce qui traîne " et de quitter les chambres. Or du fait que certains malades sont dans un état inconscient ou non locuteurs du français, l'équipe doit recourir à un accompagnant pour répondre aux questions relatives à l'évolution du « cas » (Le patient a-t-il pris tous ses repas ? A-t-il dormi ? Se serait-il plaint d'une douleur quelconque ?). Par conséquent, on assiste quotidiennement à des scènes lors desquelles, après avoir été sans complaisance mis à la porte, les accompagnants sont invités quelques instants plus tard à revenir répondre aux questions du chirurgien. Il en découle que si, pour des raisons pratiques (aération de la salle, circulation aisée de l'équipe médicale), il est important de maintenir les accompagnants en dehors des salles d'hospitalisation au moment de la ronde, leur présence est indispensable aux patients, et s'avère parfois utile aux praticiens. Ces accompagnants sont donc, ainsi que l'avait souligné Zaman (2004), un mal nécessaire. En outre, s'ils restent de façon générale dans les chambres d'hospitalisation postopératoire ou dans le pavillon « chirurgie salle », on les croise aussi bien dans les couloirs (" espace extérieur » en principe réservé aux soignants) quand ils ont besoin d'un renseignement, de précision par rapport à la posologie d'un médicament, lorsqu'un patient se plaint d'une douleur, quand une perfusion doit être remplacée. Il arrive même qu'ils soient invités jusqu'au « milieu » (« scrubbing room ») et voici pour quelles raisons.

À la pharmacie de l'hôpital où ils se rendent pour acheter les éléments de l'ordonnance, les types d'aiguilles ou de fil de suture prescrits sont régulièrement en rupture de stock. On leur délivre alors les fils et/ou aiguilles disponibles, qui ne sont pas nécessairement adaptés au type de chirurgie pratiqué (les aiguilles se cassent, les fils se rompent). Or les anesthésistes (comme la plupart des soignants) disposent de stocks de médicaments et de produits usuels, souvent vendus aux patients. Le chirurgien se tournera alors vers ses collaborateurs, invitant chacun à rechercher dans ses stocks le produit adapté, lequel sera facturé au patient. C'est pour en être informé et procéder éventuellement à une collecte urgente de fonds pour faire face à ce supplément de frais, qu'un accompagnant sera invité à se présenter devant la salle d'opération. Le mode de fonctionnement du bloc opératoire induit donc une certaine banalisation de l'espace, mais l'association du bloc opératoire à la sorcellerie, restreint l'accès à « l'extérieur » et à « l'intérieur », empêchant l'implosion de l'ensemble des frontières. 
En général, les soignants, les patients et les accompagnants qui empruntent le couloir ne s'avancent qu'exceptionnellement au-delà des cabinets d'anesthésistes ; de même, les patients ou accompagnants qui rentrent dans le local de stérilisation vont rarement au-delà. Cette attitude est manifeste même chez les soignants des autres services de l'hôpital. Nous la mettons en rapport avec une image assez particulière du bloc opératoire, associée à la sorcellerie comme on peut l'entrevoir dans cet échange bref de deux soignants que nous informions de notre désir de passer quelques nuits de garde dans le service :

SI : «Comme ça tu viendras voir les "choses” qu'il y a chez nous ».

S2 : « Un Zaïrois m'a dit m’a dit que notre bloc “n'était pas bon”. II ne le cache pas, il est dans une secte ».

SI : « Puisqu'elle ${ }^{10}$ ne connaît rien, ça va aller ».

Dans les autres services de l'hôpital, à plusieurs reprises, les soignants ont attiré notre attention sur le fait que « tous ceux qui travaillent au bloc opératoire sont des sorciers »; " il ne faut surtout pas s'y aventurer la nuit ». Des personnes précises étaient identifiées comme " sorciers » et cette accusation dépassait les frontières de l'hôpital. C'est ainsi que certains personnels du bloc opératoire relataient des rencontres avec d'anciens patients et/ou leurs accompagnants, lesquels, s'appuyant sur la consultation d'un devin, attiraient leur attention au sujet de certains collègues. Ils leur conseillaient de s'en méfier sous le prétexte qu'ils "voulaient les atteindre ». Ces personnes rappelaient que si rien n'était encore arrivé, c'est parce que les soignants visés « avaient les mains pures », c'est-à-dire qu'ils n'avaient pas eu l'intention de « nuire » à leurs patients. Nous avons ainsi recueilli des dizaines d'accusations à propos de la même personne. Les infirmiers à qui nous demandions comment ils expliquaient le fait d'être encore vivants, en dépit de «tout ce qui se passait » ont répondu : « c'est le cœur qui est pur ».

L'association du bloc opératoire à la sorcellerie mérite d'être approfondie, ainsi que l'avait aussi relevé Marie-Christine Pouchelle (2003) mettant en évidence une analogie entre espace sacré et territoire chirurgical. Cette association peut être mise en rapport avec l'exposition, qui y est quotidienne, des organes réputés être prisés par les sorciers, « mangés » en sorcellerie (le foie, le cœur). Elle participe à maintenir la plupart des personnes « extérieures » à ce service à la " périphérie ». Au final, ces éléments mettent en évidence un type spécifique d'appropriation de l'espace opératoire que, suivant la catégorisation de Katz (198I), on peut schématiser ainsi :

10 Référence à la chercheure. 
Schéma 5 : Catégorisation opérationnelle

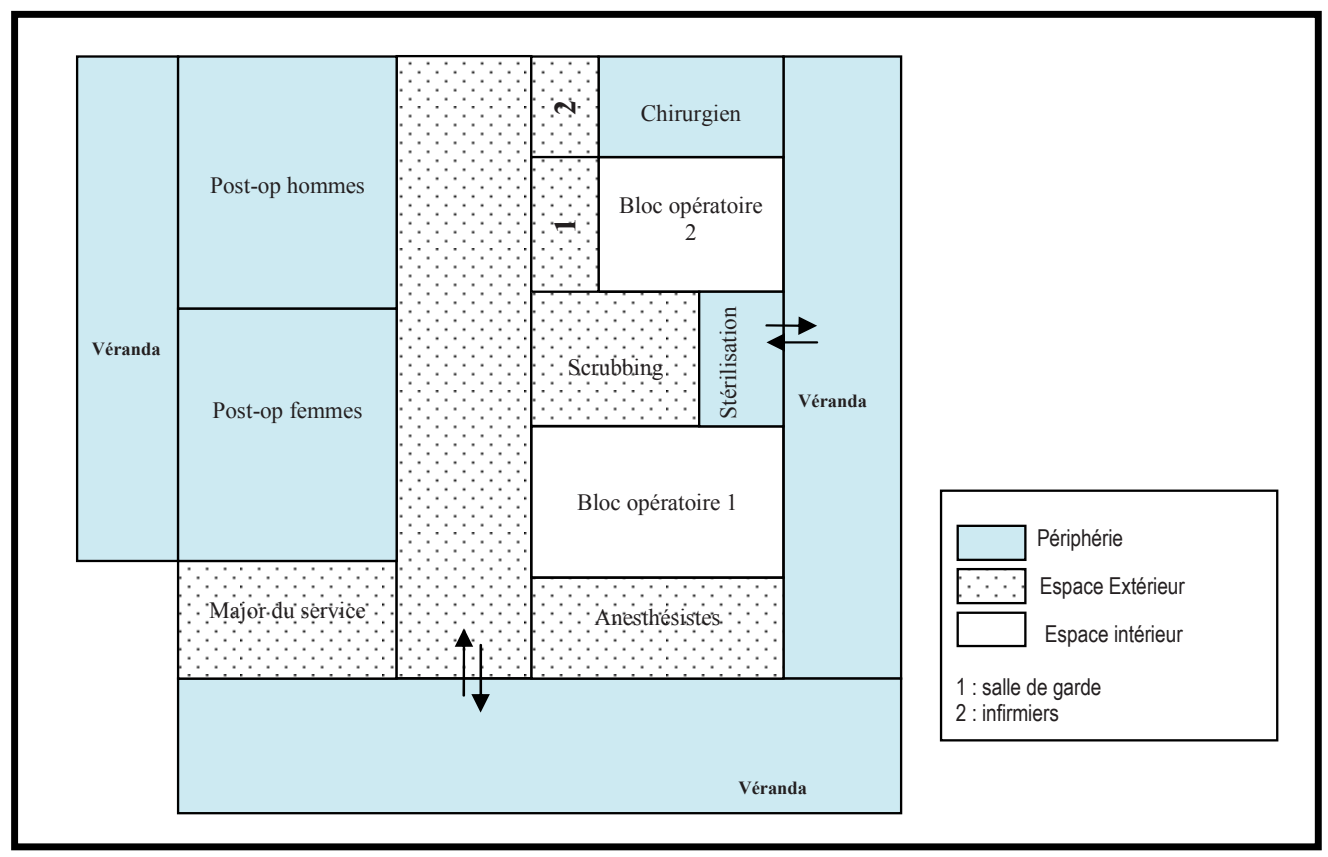

Trois catégories d'espaces (périphérie, extérieur et intérieur) donc au lieu de quatre (périphérie, extérieur, milieu et intérieur). Seront situées à la « périphérie ॥ les salles d'hospitalisations postopératoires (auxquelles ont accès les soignants, les patients ainsi que leurs accompagnants), les vérandas (espaces de sociabilité où les soignants s'installent aux heures de pause ; c'est aussi là que se retrouvent les accompagnants lorsque les patients se reposent ; ils y reçoivent la famille, jouent aux cartes et s'y allongent en attendant l'heure de dîner ou l'heure de préparer le dîner). Le cabinet du chirurgien y sera également situé. Dans « l'espace extérieur » : le local de stérilisation, le couloir de part et d'autre duquel sont les cabinets réservés aux anesthésistes, aux infirmiers, la salle de garde, le « scrubbing room » et, dans « l'espace intérieur », les salles d’opération.

Les paragraphes ci-dessus mettent en évidence un mode de fonctionnement des systèmes de santé familiers aux anthropologues étudiant l'offre de soins en Afrique. Ces travaux (pour ce qui concerne l'anthropologie française) ont mis en évidence les tensions soignants-soignés, les problèmes relatifs aux identités et rôles professionnels, les contraintes structurelles liées à la mise en place des politiques de santé (Desclaux, 1999 ; Mebtoul, $200 \mathrm{I}$; Jaffré et Olivier de Sardan, 2003 ; Vidal, Salam Fall et Gadou, 2005 ; Hane, 2007 ; Tantchou, 2007). Cependant, l'hôpital en tant que système technologique n'a pas encore été étudié dans une approche non normative. Dans cette dernière partie de notre texte, nous plaidons pour une pareille orientation et suggérons quelques pistes d'analyse pour amorcer la réflexion. 


\section{ANALYSER LES MODES D'APPROPRIATION D'UN SYSTÈME TECHNOLOGIQUE (HÔPITAL) EN AFRIQUE}

Étudier l'appropriation de l'hôpital en tant que système technologique en Afrique est périlleux; les dérives culturalistes ou racistes sont possibles. II faut savoir que l'introduction ou plutôt l'échec de l'introduction des technologies industrielles notamment en Afrique a déjà fait l'objet des travaux d'historiens. On a ainsi répété que si les gouvernements coloniaux ont importé les technologies en Afrique, celles-ci n'ont pas nécessairement été intégrées aux sociétés locales, soulevant la question de l'échec ou de l'incapacité des Africains à adopter et intégrer les technologies nouvelles. Plusieurs facteurs ont été avancés pour expliquer ce conservatisme ou cette sorte de résistance : géographique, écologique, démographique, culturel. Les facteurs culturels incluent les stratégies économiques qui mettent l'aversion du risque au-dessus de la maximisation des profits, une vision du monde qui n'encourage pas l'innovation, l'illettrisme, une certaine préférence pour les solutions politiques et militaires aux problèmes sociaux à la place des solutions économiques, la division sexuelle du travail et le mode d'éducation des enfants (Austeen et Headrick, 1983). Pour Austeen et Headrick (1983) :

« La technologie moderne est tellement dépendante des importations de gasoil et d'experts que l'introduction de l'Afrique à l'ère industrielle a été particulièrement abrupte et difficile à maîtriser » (Austeen et Headrick, 1983, p. 175).

Rapportant ces échecs au domaine de la santé publique, Reich (1988) dans un article d'une grande actualité, notait que trois types de technologie sont nécessaires pour améliorer les conditions de santé dans les pays pauvres : les mesures classiques de santé publique, les médicaments et les technologies médicales modernes. II remarquait ensuite qu'une fois implantées, ces promesses technologiques n'atteignaient pas les objectifs escomptés pour trois raisons majeures : les limites inhérentes à la technologie, les contraintes économiques relatives à leur mise en œuvre, les facteurs politiques.

« Le leurre de la solution technique est attrayant mais les questions de santé publique qui impliquent des populations et des communautés peuvent rarement être résolues par des solutions techniques. En cherchant à sauver des vies dans les pays paurres, nous devons promouvoir la production des technologies mais prendre aussi en compte la consommation des technologies, c'est-à-dire reconnaître que les effets des outils dépendent fortement de forces économiques, sociales et culturelles. L'interaction de la société avec l'environnement influence de manière décisive le contrôle de la maladie. L'efficacité de certains médicaments produits localement dans le contrôle de la 
maladie dépend donc de la mise en place de mesures adéquates de santé publique. Ainsi, la technologie est nécessaire mais non suffisante pour sauver des vies dans les pays les plus pauvres. Cette tâche doit tenir compte de la pauvreté persistante, du changement social, de l'évolution culturelle et des conflits politiques. La nouvelle technologie qu'elle soit simple ou sophistiquée est seulement l'un des éléments pour résoudre les problèmes de santé profondément inscrits dans le tissu social » (Reich, 1988, p. 92).

L'auteur appelait au développement de travaux de recherche en sciences sociales sur ces questions. II faut dire que les travaux de recherche anthropologique sur la médecine et la technologie médicale n'ont pas été entrepris de façon systématique avant le début des années 1980. Pourtant, ce sont là des points à partir desquels ont peut étudier des questions de sens, savoirs, relations de pouvoir, la culture, thèmes privilégiés de l'anthropologie. Avec l'introduction de nouvelles technologies, les pratiques, les représentations, les définitions de la santé et de la maladie, etc., sont renégociées, redéfinies ; les lieux et espaces où ces technologies sont mises en place sont réorganisés, reconfigurés de diverses manières (Casper et Koenig, 1993). Étudier les technologies médicales, les usages et les sens attribués à ces technologies fournit donc une orientation nécessairement fructueuse pour approcher la culture, la santé, la maladie, l'organisation et le changement social. Dans cette perspective, pour amorcer les travaux, du moins en ce qui concerne l'Afrique, les travaux de Hard (1993) et Grammig (2002) pourraient constituer d'excellents points de départ.

S'interrogeant sur le non-aboutissement de la plupart des coopérations technologiques, Grammig (2002) a décomposé la « technologie » en quatre composantes standards : le savoir explicite, le savoir tacite, le savoir incorporé et le savoir non incorporé. Mutuellement dépendantes, ces composantes se chevauchent et se nourrissent les unes les autres. Par ailleurs, engagé dans une coopération technologique, un individu produit de nouveaux savoirs, tacites ou explicites. Pour cet auteur, toute coopération technologique implique un échange du savoir tacite, explicite, incorporé et non incorporé. Ces quatre composantes doivent en outre être réunies pour que les résultats soient significatifs. II en conclut que la coopération pour le développement, comme la coopération technologique, a beaucoup à apprendre de la distinction entre ces composantes dans la mesure où cette coopération comprend l'utilisation d'une technologie dans un autre contexte que celui dans lequel elle a été développée. Ainsi, loin de nous demander si les sociétés africaines sont capables ou non de s'approprier des innovations ou d'innover, question ayant des fondements racistes a priori, en étudiant les technologies médicales, nous suivrons la décomposition de la technologie proposée par Grammig (2002) et poserons les questions ci-après : à quoi correspondent ces différentes composantes (le savoir explicite, le savoir tacite, le savoir incorporé et le savoir non incorporé) asso- 
ciées aux systèmes technologiques qu'est l'hôpital ? Comment sont-elles réinterprétées? Quels nouveaux savoirs tacites et explicites sont produits autour de ces technologies?

En suivant Hard (1993), nous mettrons le conflit et les rapports de pouvoir au cœur de notre analyse (cela ne ressort guère de l'ethnographie, sinon à la marge dans les relations - somme toute assez peu conflictuelles - entre les soignants et les accompagnants). Les chercheurs étudiant les controverses liées aux innovations technologiques ont mis en lumière les conflits qui naissent pendant ou après leur mise en œuvre, ainsi que les conflits manifestes dans la sphère politique. Hard (1993) suggère de traiter les conflits sociaux comme causes de l'innovation, de la diffusion, du transfert et de l'application des technologies et pas seulement comme conséquences de ces processus. Une de ses hypothèses est que les ressources et changements technologiques sont utilisés par certains groupes pour conserver ou réorganiser les relations sociales. Cela signifie que la technologie est créée, appropriée ou mise en œuvre par les groupes sociaux en situation de conflit ; dès lors, le changement technologique n'est jamais un processus socialement neutre. Hard (1993) souligne sa préférence pour le terme " conflit ॥ (à la place de controverse) qui englobe toutes sortes de luttes et oppositions sociales; conflit et pouvoir sont donc des catégories centrales dans cette approche. L'auteur fait un certain nombre de remarques puis propose des concepts clés pour mettre le conflit et le pouvoir au cœur d'une analyse de l'innovation, de l'appropriation et de la diffusion de la technologie. I) Les conflits sont de types différents : "antagonistic or unifying"|', "disintegrating or integrating"|2, "manifest or latent"|3, "conscious or subconscious"14, "direct or indirect"15. 2) La technologie et les changements technologiques peuvent être employés par certains groupes pour dominer, contrôler, exclure ou inclure d'autres groupes, pour réduire les inégalités sociales, créer du sens, sanctionner. 3) L'innovation et la diffusion des technologies peuvent être influencées par des processus de polarisation, de domination, de subordination à des niveaux macro-sociaux. Adaptée à notre objet, une pareille approche nous amènerait à nous poser les questions suivantes : qui sont les acteurs en conflit? De quelle(s) origine(s) sont-ils ? Comment se positionnent-ils les uns par rapport aux autres ? De quels types de conflits s'agit-il ? Où se situent les arènes ? Quels éléments du système technologique qu'est l'hôpital sont au cœur des conflits? Quelles sont les causes manifestes et/ou implicites de ces conflits? Quels sont les propos échangés par les protagonistes ? Est-ce que ces conflits aboutissent à une trêve ? Par quels processus ? Quelles conséquences sur l'organisation et les rapports sociaux?

II Les acteurs essaient de s'éliminer l'un l'autre de l'arène ou alors acceptent leurs adversaires comme nécessaires à la partie. Dans les deux cas, le changement technique sera utilisé pour surpasser l'adversaire.

12 Le conflit pourrait conduire à l'affaiblissement ou au renforcement des liens entre groupes opposés.

I3 Les acteurs sont ouvertement opposés ou ne permettent pas le désaccord.

14 L'arène est évidente ou non pour les acteurs concernés.

I5 Les acteurs sont directement en contact ou par l'intermédiaire des institutions sociales. 


\section{CONCLUSION}

Ce travail voulait présenter un mode spécifique d'appropriation de la technologie médicale. Nous nous sommes spécifiquement penchés sur le bloc opératoire. Nous avons présenté un bloc opératoire banalisé, où les frontières du stérile et du non-stérile, les restrictions d'accès devant faciliter son confinement et maintenir la stérilité de l'air et des espaces s'évanouissent, alors que son association à la sorcellerie entraîne la mise à distance des personnes « extérieures ॥. Nous avons précisé que, loin de proposer des analyses définitives, nous voulions réorienter les débats sur les dysfonctionnements de l'offre de soins de santé en Afrique, cela en approchant l'hôpital comme système technologique qui peut être approprié différemment, ces différences étant tributaires de l'environnement social dans lequel la technologie est diffusée. Nous avons emprunté à Austeen et Headrick (1983) la définition d'un système technologique. Pour amorcer les analyses, nous avons proposé les travaux de Grammig (2002) dont la décomposition de la «technologie » est tout à fait pertinente, et ceux de Hard (1993) qui met le conflit et les relations de pouvoir au cœur de l'analyse de l'innovation, de l'appropriation et de la diffusion des technologies.

\section{BIBLIOGRAPHIE}

Austen, R. A. et D. Headrick (1983). The Role of Technology in the African past. African Studies Review 26(3/4): 163-I84.

Beck, U. (2003). La société du risque. Sur la voie d'une autre modernité, Paris, Flammarion.

Cassel, J. (1986). Dismembering the image of god: surgeons, heroes, wimps and miracles. Anthropology Today 2(2): I3-I5.

Cassel, J. (1987). On control, certitude and the "paranoia" of surgeons. Culture, Medicine and Psychiatry I I: 229-249.

Casper, M. J. \& B. A. Koenig (1996). Reconfiguring Nature and Culture: Intersections of Medical Anthropology and Technoscience Studies. Medical Anthropology Quarterly 10(4): 523-536.

Chen, L. et P. Hanvoravongchai (2005). HIVIAIDS and human resources. Bulletin of the World Health Organization 83(4): 243.

Beyeme Ondoua, J.-P. (2002). Le système de santé camerounais. ADSP(39): 6I-65.

Desclaux, A. (1999). Des infirmières face au sida. Impact de l'épidémie sur les rôles professionnels dans un service de pédiatrie du Burkina Faso. In C. Becker, J.-P. Dozon, C. Obbo et M. Touré. Vivre et penser le sida en Afrique. Paris, Dakar, Karthala/Codesria/ IRD: P54I-555.

Delanyo, D. (2005). Wastage in the health workforce: some perspective from African countries. Human Resources for Health 3(6): |25I-I26I.

Hirschauer, S. (1991). The manufacture of bodies in surgery. Social Studies of Science 21 (2): 279-3I 9.

Hane, F. (2007). Émergence de la fonction soignante. Reconfigurations professionnelles et politiques publiques autour de la prise en charge de la tuberculose au Sénégal. Marseille, EHESS, Thèse de doctorat : 372 . 
Hard, M. (1993). Beyond Harmony and Consensus: A Social Conflict Approach to Technology. Science, Technology, \& Human Values 18(4): 408-432.

Israr, S. (2000). Coping strategies of health personnel during economic crisis: a case study from Cameroon. Tropical Medicine and International Health 5(4): 288-292.

Jaffré, Y. et J.-P. Olivier de Sardan (2003). Une médecine inhospitalière. Les difficiles relations entre soignants et soignés dans cinq capitales d'Afrique de l'Ouest. Paris, Marseille, ApadKarthala.

Kushner, A. L., S. J. Mannion, et al. (2004). Secondary crisis in African health care. The Lancet 363(|4|9): 1478.

Layne, L. L. (2000). The Cultural Fix: An Anthropological Contribution to Science and Technology Studies. Science, Technology, \& Human Values 25(3): 352-379.

Marchal, B. et V. De Brouwere (2004). Global human resources crisis. The Lancet 363: 2191-2192.

Mebtoul, M. (200I). Les acteurs sociaux face à la santé publique : médecins, État et usagers (Algérie), Les Professionnels de santé. Réseau Anthropologie de la santé (Bulletin, $\left.\mathrm{n}^{\circ} 2\right)$ : $155-164$.

Muula, A. (2005). Is there any solution to the brain drain of health professionals and knowledge from Africa? Croat Med J._46(I): 21 -9.

Narasimhan, V. et al. (2004). Responding to the global human resources crisis. The Lancet 363: I469-I 472.

Pollock, D. (1995). Masks and the semiotics of identity. The Journal of the Royal Anthropological Institute I(3): 58I-597.

Pouchelle, M. C. (2007). Quelques touches hospitalières. Terrain(49): I I-26.

Raviola, G., M. Machoki, et al. (2002). HIV, disease plague, demoralization and burnout: resident experience of the medical profession in Nairobi, Kenya. Culture Medicine Psychiatry 26(I): 55-86.

Reich, M. R. (1988). Technical Fixes and Other Problems in Saving Lives in the World's Poorest Countries. Journal of Public Health Policy 9(I): 92- 103.

Sartorius, N. (2005). An epidemic is threatening health care worldwide. Croat Med J. 46(I): 1452-153.

Tantchou, J. (2007). Epidémie et politique en Afrique. Maladie du sommeil et tuberculose au Cameroun. Paris, L'Harmattan.

Vassy, C. (1999). Travailler à l'hôpital en Europe. Apport des comparaisons internationales à la sociologie des organisations. Revue Française de sociologie 40(2): 325-356.

Vayre, P. (2000). Gestion des risques en pratique chirurgicale : la sérenité retrouvée. Annales de chirurgie 125: 925-928.

Vidal L., Salam Fall A. et Gadou D. (2005). Les Professionnels de santé en Afrique de l'Ouest, entre savoirs et pratiques : paludisme, tuberculose et prévention au Sénégal et en Côte d'lvoire, Paris : L'Harmattan, $328 \mathrm{p}$.

Zaman, S. (2004). Poverty and violence, frustration and inventiveness: hospital ward life in Bangladesh. Social Science and Medicine 59: 2025-2036. 
Josiane TANTCHOU est anthropologue, docteure de l'École des Hautes Études en Sciences Sociales (EHESS) de Paris. Ses recherches ont porté sur les politiques et les systèmes de santé en Afrique, avec un ancrage historique. Elle s'est notamment intéressée aux politiques de lutte contre la tuberculose, la maladie du sommeil et le $\mathrm{VIH} /$ sida. Ces dernières années, elle s'est penchée sur les relations entre les conditions de travail des soignants et le "burn-out ", syndrome d'épuisement professionnel du soignant et l'appropriation des technologies médicales. Bureau d'études et de recherches qualitatives (Bureq)

$\begin{array}{ll}\text { ADRESSE : } & \text { I5, rue Pierre Dupré } \\ & \text { F-I3008 Marseille } \\ & \text { Tél. : +3349I } 58 \text { II } 57 \\ & \text { Fax : +3349I } 58 \text { II } 57 \\ \text { COURRIEL : } & \text { Yakam.Tantchou@orange.fr }\end{array}$

Marc-Éric GRUÉNAIS est anthropologue, directeur de recherche à l'Institut de Recherche pour le Développement (IRD), responsable de l'équipe « Maladies Transmissibles, Systèmes de santé, Sociétés » (UMR 912 - INSERM/IRD/U2) et enseignant-chercheur à l'Institut National d'Administration Sanitaire (Rabat) ; il est également chargé de cours au département d'anthropologie de l'Université de Provence (Aix-en-Provence). Ses principaux travaux ont trait aux professionnels de santé, à l'organisation locale des systèmes de santé, à la qualité des soins, à la santé reproductive (mortalité maternelle et $\mathrm{VIH} /$ sida) et au paludisme ; ils portent sur le Maroc, le Burkina Faso et le Cameroun.

ADRESSE : $\quad$ UMR 912 (INSERM-IRD-U2), Sciences Économiques et Sociales, Systèmes de santé, Sociétés Observatoire Régional de la Santé (PACA)

23, rue Stanislas Torrents

F-I 3006 Marseille

COURRIEL : $\quad$ Marc-Eric.Gruenais@ird.fr 


\section{ABSTRACT: BLURRING BOUNDARIES: THE APPROPRIATION OF THE SURGICAL AREA IN A CONTEXT OF HEALTH SYSTEM CRISIS}

Anthropological research on surgical practice is rare. Joan Cassel (1986, 1987) studied the surgeon's personality in relation with the specificities of surgery as a specialty. Marie-Christine Pouchelle (2003) analyzed the "touch" in the hospital, with a special emphasis on its surgical aspects. Pearl Katz ( $198 \mathrm{I}$ ) proposed a typology of the operating theater according to the criteria of sterile and non sterile. Hirschauer (I99I) analyzed the process of surgery as an encounter of two disciplined bodies: the patient's body and the surgeon's body. We follow Katz (198I) analysis and propose a reflection on how, in the context of a health system crisis, the operating theater and its equipment are appropriated.We show that, in this context, restrictions that make the operating theater a confined space are blown apart, the boundaries of the sterile and non sterile vanish, the surgical practice is stripped of its thickness, and the surgical area is trivialized. Far from proposing a final analysis, this article refocuses the debate on the African health systems failures by approaching the surgical theater as part of a technological system, the hospital, which can be appropriated in various ways depending on the socioeconomic context.

KEYWORDS : health anthropology, medicine, surgery, sterile, boundaries, hospital, technological system, Cameroun, Africa.

\section{RESUMEN: LAS FRONTERAS BORROSAS DEL ESTERIL Y DEL NO ESTERIL : EL USO DEL BLOQUE OPERATORIO EN UN SISTEMA DE SALUD EN CRISIS (CAMERUN)}

Los trabajos antropológicos sobre los espacios operatorios, la pràctica de la cirurgía o la profesión de cirujano son poco frecuentes (Hirschauer, 1991). Joan Cassel (Cassel, 1986, 1987) se ha interesado a la personalidad de los cirurjanos en ralación con las especificidades de la cirurjía como especialización. MarieChristine Pouchelle (2003) ha analizado el « toque » en el hospital, insistiendo particularmente sobre la dimensión cirurjical. Pearl Katz a propuesto una tipología del boque operatorio en función de los criterios del etéril y del no estéril que se aplican y ha obseravdo mlos rituales y procedimientos técnicos en los bloques operatorios. Hirschauer (I99I) se interesó por el acto cirurjical como momento de encuentro entre dos cuerpos disciplinados (disciplined bodies): el corps del paciente (parcelled patient-body) y el « cuerpo cirurjical ». Hemos querido retomar las reflexiones de Katz (I98I) y sobre todo proponer una reflexión en la manera que el bloque operatorio y sus equipos se estan apropriando, y cómo su uso se está desviando o re-negociado, en el contexto de crisis del sistema de salud. Mostramos que esta manera de apropropriarse del boque operatorio rompe las restricciones que 
hacen de él un espacio confinado, estéril; los límites del estéril y no estéril se desvanecen, la práctica cirurjical se deshace de su espesor, el bloque se banaliza. Lejos de proponer un análisis definitivo, este artículo quiere reorientar los debates sobre disfuncionamientos de los sistemas de salud en Africa. Para hacer eso, proponemos de examinar el espacio operatorio como elemento de un sistema tecnológico: el hospital. Este enfoque nos permitirá ir más allá de las orientaciones normativas usuales en antropología de la enfermedad para proponer una lectura del modo organizativo y de funcionamiento de las estructuras hospitalarias en Africa como tipo específico de apropriación del sistema tecnológico que se debe decriptar y analizar.

PALABRAS CLAVES : antropologia de la salud, cirurjia, practica cirurjical, esteril, fronteras, hospital, sistema tecnologico, Camerun, Africa. 